\section{Associação entre o uso de sais de ferro durante a gestação e nascimento pré-termo, baixo peso ao nascer e muito baixo peso ao nascer}

\author{
Association between iron supplementation during \\ pregnancy and prematurity, low birth weight, \\ and very low birth weight
}

\footnotetext{
1 Faculdade de Farmácia, Universidade Federal do Rio Grande do Sul, Porto Alegre, Brasil. 2 Faculdade de Medicina, Universidade Federal do Rio Grande do Sul, Porto Alegre, Brasil.

Correspondência T. S. Dal Pizzol

Departamento de Produção e Controle de Medicamentos Faculdade de Farmácia, Universidade Federal do Rio Grande do Sul.

Rua Vicente da Fontoura 2035, apto. 602, Porto Alegre, RS 90640-003, Brasil. tatiane.silva@ufrgs.br
}

\begin{abstract}
The objective was to evaluate the association between prophylactic iron supplementation and prematurity and low birth weight. Pregnant women 20 years and older with 21 to 28 weeks of gestational age were enrolled consecutively in prenatal services in the Unified National Health System in six Brazilian State capitals between 1991 and 1995. Socio-demographic data and information on iron supplementation up until the 28 th gestational week were obtained by means of an interview. Outcomes and other variables were collected from medical records. Anemia was present in $31.3 \%$ of the 3,865 women. Among anemic women (hemoglobin < 11.0g/dL), 29.8\% were taking iron supplements, as compared to $16.7 \%$ among non-anemic women (hemoglobin $\geq 11.0 \mathrm{~g} / \mathrm{dL}$ ). After adjusting for potential confounders, iron was not associated with prematurity $(O R=0.88 ; 95 \% C I: 0.73-1.07)$, low birth weight (OR $=0.99 ; 95 \%$ CI: 0.75-1.31), or very low birth weight (OR=0.58; 95\%CI: 0.29-1.13). The results suggest that iron supplementation up to the 28th gestational week does not reduce the risk of prematurity, low birth weight, or very low birth weight.
\end{abstract}

Iron Salts; Pregnancy; Premature Infant; Birth Weight
Tatiane da Silva Dal Pizzol 1,2

Elsa Regina Justo Giugliani 2

Sotero Serrate Mengue 2

\section{Introdução}

A anemia por deficiência de ferro constitui a carência nutricional mais comum no mundo, afetando principalmente crianças pequenas e mulheres em idade fértil, incluindo gestantes 1,2. No Brasil, estima-se que de cada dez gestantes que fazem o pré-natal, três são anêmicas ${ }^{3}$.

A anemia na gestação representa uma questão de interesse à saúde pública pelo provável aumento no risco de morbimortalidade perinatal. Revisão sistemática de dez estudos observacionais publicados em língua inglesa e realizados nos Estados Unidos (4), Reino Unido (1), Holanda (2), China (2) e Zaire (1), sugere um risco levemente aumentado de nascimento pré-termo para anemia diagnosticada em mulheres com menos de vinte semanas de gestação, mas não em gestantes com mais de trinta semanas. Em relação ao peso ao nascer, não foi verificada associação com anemia no início da gestação; entretanto, para anemia no final da gestação foi verificada uma associação negativa (razão de chances - RC = 0,80; intervalo de 95\% de confiança - IC95\%: 0,65-0,99) 4. Outra revisão sistemática que incluiu estudos publicados em espanhol e francês, além do inglês, concluiu que as evidências provenientes de estudos com delineamento apropriado sobre a relação causal entre anemia moderada ou grave e os desfechos nascimento pré-termo ou baixo peso ao nascer são inconsistentes 5 . 
A Organização Mundial da Saúde (OMS) e o Ministério da Saúde recomendam a suplementação diária de $60 \mathrm{mg}$ de ferro elementar a todas as gestantes na segunda metade da gravidez, independente do diagnóstico de anemia ${ }^{2,6}$. Apesar dessa recomendação, existe uma tendência mundial de reduzir a quantidade de ferro profilático, seja pelo freqüente abandono devido aos efeitos adversos, seja pela absorção reduzida do ferro com os esquemas diários e de altas doses 7. Nesse sentido, ensaios clínicos têm comparado diferentes esquemas posológicos de suplementação com ferro 8,9,10,11.

As evidências sobre a eficácia dos sais de ferro na melhora dos índices hematológicos em gestantes são fortes 12. Por outro lado, as informações sobre os benefícios clínicos para a mãe ou o recém-nascido advindos do uso de ferro durante a gestação, tanto em mulheres anêmicas quanto em mulheres não-anêmicas são insuficientes, conforme verificado em revisões sistemáticas de ensaios clínicos controlados 1,12. Ensaios clínicos que avaliaram o efeito da suplementação de ferro na incidência de nascimento pré-termo e baixo peso ao nascer não encontraram qualquer efeito benéfico decorrente da administração profilática de ferro durante a gestação 8,9,10,11. Poucos estudos observacionais sobre a eficácia dos sais de ferro em desfechos clínicos perinatais foram localizados na literatura, sendo que a maioria tem enfocado o uso de ferro combinado a outros minerais e vitaminas.

Com o propósito de investigar os benefícios clínicos da administração de sais de ferro em gestantes brasileiras em condições ambulatoriais usuais, o presente estudo avaliou a associação entre utilização de ferro profilático ou terapêutico e nascimento pré-termo, baixo peso ao nascer ou muito baixo peso ao nascer em serviços de acompanhamento pré-natal de seis capitais brasileiras, no período de 1991 a 1995.

\section{Métodos}

Os dados do presente trabalho foram obtidos de uma coorte multicêntrica, que investigou diabetes e intolerância à glicose em grávidas, em relação à prevalência, fatores de risco, incidência de complicações obstétricas e neonatais, fatores prognósticos e critérios diagnósticos. A primeira fase do estudo incluiu o arrolamento das gestantes, realização da entrevista, aferição de medidas antropométricas e de tolerância à glicose. Na segunda fase, foi realizado o acompanhamento da gestação, por meio da revisão dos prontuários, até o parto. A terceira fase foi composta pelas informações sobre o parto e o recém-nascido.
A amostra foi constituída por gestantes que consecutivamente procuravam atendimento em ambulatórios de acompanhamento pré-natal ligados ao Sistema Único de Saúde (SUS) de Porto Alegre (Rio Grande do Sul), São Paulo, Rio de Janeiro, Salvador (Bahia), Fortaleza (Ceará) e Manaus (Amazonas), entre fevereiro de 1991 e junho de 1995. Os critérios para o arrolamento das gestantes foram: idade de 20 anos ou mais, idade gestacional entre 21 e 28 semanas e, não ser portadora de diabetes antes da gravidez.

As informações relacionadas com os critérios de inclusão foram obtidas do prontuário da gestante ou da carteira do pré-natal. Nas ocasiões em que a consulta era a primeira do pré-natal, os dados podiam ser obtidos diretamente com a gestante.

As características sócio-demográficas das pacientes foram obtidas por intermédio da entrevista realizada na primeira fase do estudo. $\mathrm{O}$ uso de sais de ferro declarado foi registrado por meio de perguntas abertas e fármaco-direcionadas incluídas na entrevista, quando as gestantes encontravam-se entre a 21a e 28a semanas de gestação. A identificação das substâncias ativas presentes em cada um dos produtos referidos pelas gestantes foi feita pela consulta ao Catálogo Brasileiro de Especialidades Farmacêuticas, Dicionário de Especialidades Farmacêuticas (edições de 1984 a 1995) 13,14,15,16,17,18,19,20,21,22,23,24 e bulas dos medicamentos, quando necessário. A pergunta "A Sra. está tomando vitaminas, ferro ou flúor durante esta gravidez?”, permitiu a declaração de uso de produtos contendo sais de ferro isolados ou associados a vitaminas, em uma mesma forma farmacêutica ou em formas farmacêuticas separadas. No presente estudo foi avaliado apenas o uso de ferro isolado administrado até a 28a semana de gestação. Não foram coletadas informações sobre a duração do uso de ferro.

Os principais eventos perinatais analisados foram coletados com base em registros disponíveis no prontuário da mãe e do neonato. Baixo peso ao nascer foi definido para todo recém-nascido com menos de $2.500 \mathrm{~g}$, considerando-se a primeira medida de peso do recém-nascido obtida após o nascimento. O mesmo registro foi utilizado para muito baixo peso ao nascer, definido como todo recém-nascido com menos de 1.500g. O nascimento pré-termo foi definido para todo recém-nascido com menos de 37 semanas completas de gestação. Para a definição da idade gestacional foi utilizado um critério hierarquizado, previamente descrito 25 , baseado em quatro parâmetros: ecografia obstétrica, avaliação da idade gestacional neonatal (método Capurro), data da última menstruação e última altura uterina. 
Gestantes com valor de hemoglobina inferior a 11,0g/dL foram classificadas como anêmicas, e aquelas com valor igual ou acima de $11,0 \mathrm{~g} / \mathrm{dL}$ foram classificadas como não-anêmicas, em concordância com as recomendações da OMS e do Ministério da Saúde. Os dados laboratoriais foram obtidos do prontuário da gestante na segunda fase do estudo, período em que ela se encontrava entre o segundo e terceiro trimestres de gravidez. Quando a paciente apresentava mais de uma medida de hemoglobina, considerava-se o resultado mais baixo.

A associação entre uso de ferro e ocorrência de eventos adversos no neonato foi expressa pela RC. Para estimar a significância estatística das associações foi aplicado o teste do qui-quadrado, e os resultados foram apresentados em IC95\%. Para as estimativas da RC ajustada para potenciais confundidores relatados na literatura, foi desenvolvida modelagem por regressão de Poisson com ajuste robusto da variância. Foram examinados vários fatores de risco para nascimento pré-termo, previamente descritos na literatura, incluindo características sócio-demográficas (idade, altura, cor da pele, escolaridade, centro de realização da pesquisa, situação conjugal e índice de massa corporal - IMC - pré-gestacional), história reprodutiva (aborto prévio e planejamento da gravidez), intercorrências na gestação (diabetes gestacional, distúrbios hipertensivos, anemia, infecção materna e hemorragia anteparto) e outros (número de consultas pré-natais, tipo de parto, tabagismo e anomalia congênita). Para os desfechos baixo peso e muito baixo peso ao nascer, foram testadas as seguintes co-variáveis, além das já citadas: paridade, idade gestacional na primeira consulta, sexo do recém-nascido e parto pré-termo. O centro de realização da pesquisa foi incorporado ao modelo para ajustar as diferenças regionais em relação às características sócio-demográficas das gestantes e à freqüência de uso de antianêmicos, conforme descrito em publicação anterior 26 .

Os procedimentos de análise foram utilizados para toda a amostra e separadamente nos estratos formados pelos níveis de hemoglobina $<11,0 \mathrm{~g} / \mathrm{dLe} \geq 11,0 \mathrm{~g} / \mathrm{dL}$, a fim de verificar possíveis diferenças na efetividade dos sais de ferro utilizados em condições profiláticas ou terapêuticas.

Os dados foram armazenados usando-se o programa Epi Info versão 6.04b (Centers for Disease Control and Prevention, Atlanta, Estados Unidos) e analisados no programa SAS 9.1 (SAS Inst., Cary, Estados Unidos).

O estudo original foi considerado de risco mínimo, por envolver apenas a realização de uma entrevista e de medidas antropométricas, afora o teste de tolerância à glicose. Os dados oriundos dos questionários, objeto de estudo desta análise secundária, foram tratados dentro do princípio da confidencialidade.

\section{Resultados}

De uma amostra total de 5.564 gestantes, 3.865 apresentaram registros laboratoriais de hemoglobina, constituindo a amostra inicial analisada. As gestantes excluídas $(n=1.699)$ apresentaram diferenças nas seguintes características em relação às analisadas: cor da pele branca, mais idade, maior escolaridade, menor número de filhos e de consultas pré-natal, e atendimento em São Paulo. As análises preliminares para nascimento pré-termo incluíram 3.477 gestantes. Os 388 casos excluídos pela ausência de registro da idade gestacional ao nascimento apresentavam menor número de filhos, não possuíam companheiro e também eram provenientes de São Paulo. Para as análises de baixo peso ou muito baixo peso ao nascer foram analisados 3.404 casos, sendo excluídos 461 sem registro do peso do recém-nascido, também caracterizados por apresentarem menor número de filhos, ausência de companheiro e atendimento em São Paulo.

As principais características sócio-demográficas e clínicas das gestantes analisadas são apresentadas na Tabela 1. Em relação às gestantes não-anêmicas, as anêmicas apresentaram com maior freqüência: cor da pele não-branca, menor escolaridade, maior número de filhos e baixo peso pré-gestacional. Além disso, iniciaram o prénatal mais tarde e fizeram menos consultas de pré-natal que as gestantes não-anêmicas.

Das gestantes pesquisadas, o uso declarado de sais de ferro foi verificado para 805 mulheres $(20,8 \%)$. A prevalência de anemia na amostra analisada foi de $31,3 \%$, considerando-se o ponto de corte da hemoglobina em 11,0g/dL. A Tabela 2 mostra o uso de sais de ferro, de acordo com a prevalência de anemia. Entre as gestantes anêmicas, $29,8 \%$ utilizavam ferro, contra $16,7 \%$ entre as não-anêmicas, com uma diferença estatisticamente significativa $(\mathrm{p}<0,01)$.

A Tabela 3 apresenta as RC não-ajustadas e ajustadas para os desfechos analisados. Foi verificada uma associação positiva entre ferro e nascimento pré-termo na análise bruta $(\mathrm{RC}=1,33$; IC95\%: 1,08-1,65), mas não no modelo ajustado para escolaridade da mãe, centro de realização da pesquisa, número de consultas pré-natal, IMC pré-gestacional, distúrbios hipertensivos e hemorragia anteparto ( $\mathrm{RC}=0,88$; IC95\%: 0,73-1,07). Quando as gestantes foram analisadas de acordo com os níveis de hemoglobina, a ausência de associação se manteve. 
Características sócio-demográficas e clínicas das 3.865 gestantes, de acordo com os índices de hemoglobina (Hb). Estudo Brasileiro sobre Diabetes Gestacional, 1991 a 1995.

\begin{tabular}{|c|c|c|c|c|}
\hline \multirow[t]{2}{*}{ Características } & \multicolumn{2}{|c|}{$\begin{array}{c}\text { Anêmicas } \\
(\mathrm{Hb}<11,0 \mathrm{~g} / \mathrm{dL})\end{array}$} & \multicolumn{2}{|c|}{$\begin{array}{l}\text { Não-anêmicas } \\
\text { (Hb } \geq 11,0 \mathrm{~g} / \mathrm{dL} \text { ) }\end{array}$} \\
\hline & $\mathrm{n}$ & $\%$ & $\mathrm{n}$ & $\%$ \\
\hline \multicolumn{5}{|l|}{ Idade (anos) } \\
\hline $20-34$ & 1.073 & 88,8 & 2.351 & 88,5 \\
\hline $35-48$ & 135 & 11,2 & 306 & 11,5 \\
\hline \multicolumn{5}{|l|}{ Cor da pele * } \\
\hline Branca & 447 & 37,0 & 1.121 & 42,2 \\
\hline Não-branca & 761 & 63,0 & 1.536 & 57,8 \\
\hline \multicolumn{5}{|l|}{ Escolaridade (anos) ** } \\
\hline$<8$ & 645 & 53,4 & 1.232 & 46,4 \\
\hline$\geq 8$ & 563 & 46,6 & 1.425 & 53,6 \\
\hline \multicolumn{5}{|l|}{ Número de filhos ** } \\
\hline 0 & 271 & 24,6 & 718 & 30,9 \\
\hline 1 & 365 & 33,2 & 786 & 33,8 \\
\hline$\geq 2$ & 464 & 42,2 & 819 & 35,3 \\
\hline \multicolumn{5}{|l|}{ Situação conjugal } \\
\hline Com companheiro & 1.098 & 90,9 & 2.455 & 92,5 \\
\hline Sem companheiro & 110 & 9,1 & 198 & 7,5 \\
\hline \multicolumn{5}{|c|}{ IMC pré-gestacional $\left(\mathrm{kg} / \mathrm{m}^{2}\right)$ ** } \\
\hline$<19,8$ & 226 & 19,8 & 395 & 15,5 \\
\hline $19,8-26,0$ & 727 & 63,6 & 1.612 & 63,4 \\
\hline $26,1-29,0$ & 116 & 10,1 & 302 & 11,9 \\
\hline$>29,0$ & 74 & 6,5 & 234 & 9,2 \\
\hline \multicolumn{5}{|c|}{ Idade gestacional na primeira consulta (semanas) ** } \\
\hline$\leq 13$ & 258 & 24,4 & 837 & 35,0 \\
\hline $13-26$ & 655 & 62,0 & 1.336 & 55,8 \\
\hline$>26$ & 143 & 13,5 & 220 & 9,2 \\
\hline \multicolumn{5}{|c|}{ Número de consultas pré-natal ** } \\
\hline $0-5$ & 651 & 61,6 & 1.104 & 46,1 \\
\hline 6 ou mais & 405 & 38,4 & 1.289 & 53,9 \\
\hline \multicolumn{5}{|c|}{ Centro de realização da pesquisa } \\
\hline Porto Alegre & 227 & 18,8 & 610 & 23,0 \\
\hline Salvador & 160 & 13,2 & 615 & 23,1 \\
\hline Fortaleza & 448 & 37,1 & 479 & 18,0 \\
\hline Rio de Janeiro & 178 & 14,7 & 242 & 9,1 \\
\hline São Paulo & 85 & 7,0 & 414 & 15,6 \\
\hline Manaus & 110 & 9,1 & 297 & 11,2 \\
\hline 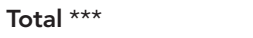 & 1.208 & 100,0 & 2.657 & 100,0 \\
\hline
\end{tabular}

IMC: índice de massa corporal.

${ }^{*} p<0,01$;

** $\mathrm{p}<0,001$

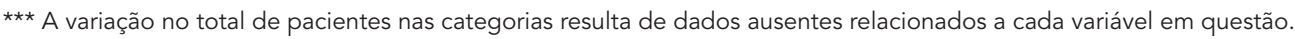

Em relação ao peso ao nascer, a Tabela 3 mostra ausência de associação estatisticamente significativa nas análises brutas (baixo peso ao nascer: $\mathrm{RC}=1,11$; IC95\%: 0,83-1,48; muito baixo peso ao nascer: $\mathrm{RC}=1,42$; IC95\%: 0,76-2,63) e nos modelos ajustados para potenciais confundidores (baixo peso ao nascer: $\mathrm{RC}=0,99$; IC95\%: $0,75-1,31$; muito baixo peso ao nascer: $\mathrm{RC}=0,58$; IC95\%: 0,29-1,13). 
Uso de sais de ferro, de acordo com a prevalência de anemia. Estudo Brasileiro sobre Diabetes Gestacional, 1991 a 1995.

\begin{tabular}{lcccc}
\hline Uso & Gestantes com anemia $(\mathrm{Hg}<11,0 \mathrm{~g} / \mathrm{dL})$ & Gestantes sem anemia $(\mathrm{Hg} \geq 11,0 \mathrm{~g} / \mathrm{dL})$ \\
$\%$ & $\mathbf{n}$ & $\mathbf{n}$ & \\
\hline Sim & 360 & 29,8 & 445 & 16,7 \\
Não & 848 & 70,2 & 2.212 & 83,3 \\
Total & 1.208 & 100,0 & 2.657 & 100,0 \\
\hline
\end{tabular}

$* p<0,01$

Razão de chances (RC) não ajustada e ajustada para nascimento pré-termo, baixo peso ao nascer e muito baixo peso ao nascer, de acordo com o uso de sais de ferro. Estudo Brasileiro sobre Diabetes Gestacional, 1991 a 1995.

\begin{tabular}{|c|c|c|c|c|c|c|c|c|}
\hline & \multicolumn{4}{|c|}{ Uso de sais de ferro } & \multicolumn{2}{|c|}{ Todas as gestantes } & \multirow{3}{*}{$\begin{array}{c}\text { Gestantes com } \\
\text { anemia } \\
\text { n }=1.029 \\
\text { RC ajustada } \\
\text { (IC95\%) }\end{array}$} & \multirow{3}{*}{$\begin{array}{c}\text { Gestantes sem } \\
\text { anemia } \\
\text { n }=2.319 \\
\text { RC ajustada } \\
\text { (IC95\%) }\end{array}$} \\
\hline & \multicolumn{2}{|c|}{ Usou } & \multicolumn{2}{|c|}{ Não usou } & \multirow{2}{*}{$\begin{array}{c}\mathrm{n}=3.477 \\
\mathrm{RC} \text { não ajustada } \\
\text { (IC95\%) }\end{array}$} & \multirow{2}{*}{$\begin{array}{c}\mathrm{n}=3.303 \\
\mathrm{RC} \text { ajustada } \\
\text { (IC95\%) }\end{array}$} & & \\
\hline & $\mathrm{n} /$ Total & $\%$ & $\mathrm{n} /$ Total & $\%$ & & & & \\
\hline Nascimento pré-termo & $138 / 725$ & 19 & $412 / 2.752$ & 15 & $\begin{array}{c}1,33 \\
(1,08-1,65)\end{array}$ & $\begin{array}{c}0,88 \\
(0,73-1,07) \text { * }\end{array}$ & $\begin{array}{c}0,90 \\
(0,68-1,20) \star \star\end{array}$ & $\begin{array}{c}0,91 \\
(0,70-1,17) * \star\end{array}$ \\
\hline Baixo peso ao nascer & $66 / 706$ & 9,3 & $229 / 2.698$ & 8,5 & $\begin{array}{c}1,11 \\
(0,83-1,48)\end{array}$ & $\begin{array}{c}0,99 \\
(0,75-1,31) * \star \star\end{array}$ & $\begin{array}{c}0,83 \\
(0,54-1,26) \#\end{array}$ & $\begin{array}{c}1,09 \\
(0,75-1,57) \#\end{array}$ \\
\hline Muito baixo peso ao nascer & $14 / 706$ & 2,0 & $38 / 2.698$ & 1,4 & $\begin{array}{c}1,42 \\
(0,76-2,63)\end{array}$ & $\begin{array}{c}0,58 \\
(0,29-1,13) \# \#\end{array}$ & $\begin{array}{c}0,73 \\
(0,26-2,08) \# \#\end{array}$ & $\begin{array}{c}0,50 \\
(0,20-1,22) \# \# \#\end{array}$ \\
\hline
\end{tabular}

* Ajustado para: escolaridade da mãe, centro de realização da pesquisa, número de consultas pré-natal, índice de massa corporal (IMC) pré-gestacional, distúrbios hipertensivos e hemorragia anteparto;

** Ajustado para: escolaridade da mãe, centro de realização da pesquisa, número de consultas pré-natal, distúrbios hipertensivos, hemorragia anteparto e IMC pré-gestacional;

*** Ajustado para: estatura da mãe, centro de realização da pesquisa, nascimento pré-termo, distúrbios hipertensivos, hemorragia anteparto, tabagismo, IMC pré-gestacional, anomalia congênita e número de consultas pré-natal;

\# Ajustado para: centro de realização da pesquisa, nascimento pré-termo, distúrbios hipertensivos, hemorragia anteparto, tabagismo e número de consultas pré-natal;

\#\# Ajustado para: centro de realização da pesquisa, distúrbios hipertensivos, hemorragia anteparto, número de consultas pré-natal e anomalia congênita;

\#\#\# Ajustado para: centro de realização da pesquisa, distúrbios hipertensivos, hemorragia anteparto e número de consultas pré-natal.

\section{Discussão}

A prevalência de anemia nas gestantes atendidas pelo SUS em seis capitais brasileiras $(31,3 \%)$ incluídas nesta análise confirma a média estimada pelo Ministério da Saúde para anemia na gravidez. Entre as gestantes anêmicas, 29,8\% utilizavam sais de ferro; entre as não-anêmicas o percentual foi de 16,7. Aliadas a esses resultados, as diferenças entre as gestantes anêmicas e não-anêmicas, apresentadas na Tabela 1, sugerem que as gestantes anêmicas pertencem a um grupo de menor nível sócio-econômico que utili- za sais de ferro como uma intervenção predominantemente terapêutica, conforme já apontado anteriormente por Mengue et al. 27 .

$\mathrm{Na}$ amostra analisada, nenhuma associação foi verificada entre ferro e desfechos adversos no resultado da gravidez, tanto em gestantes com níveis normais de hemoglobina quanto em gestantes com níveis de hemoglobina inferior a $11,0 \mathrm{~g} / \mathrm{dL}$.

Os estudos localizados na literatura divergem quanto aos efeitos do ferro no nascimento pré-termo em gestantes anêmicas. Macgregor 28, em estudo observacional realizado no Quênia, 
comparou a freqüência de prematuridade entre 153 gestantes tratadas com sulfato ferroso até a 36a semana de gestação e 448 não-tratadas, todas com diagnóstico de anemia moderada ou grave e hospitalizadas para o trabalho de parto. A taxa de nascimentos prematuros foi de $35 \%$ no grupo não-tratado e de $9 \%$ no grupo tratado. Nenhum tipo de tratamento estatístico foi utilizado para controle de potenciais fatores de confusão. Hemminki \& Rimpelä 29 , comparando o efeito do uso terapêutico com o uso profilático de ferro, em um ensaio clínico com 2.912 gestantes finlandesas, não verificaram diferenças no percentual de recém-nascidos prematuros entre os dois grupos analisados. Em uma revisão sistemática de 17 ensaios clínicos controlados, incluindo $2.578 \mathrm{mu}$ lheres, Reveiz et al. ${ }^{1}$ verificaram que em nenhum dos ensaios clínicos que compararam ferro com placebo foi avaliado o efeito do tratamento antianêmico no resultado da gravidez, especificamente em desfechos clínicos relevantes como parto prematuro e baixo peso ao nascer. Tendo em vista que a anemia ferropriva tem sido apontada por muitos autores como um fator de risco para nascimento pré-termo, conforme verificado em revisão sistemática 4 , o tratamento da anemia com ferro poderia reduzir a incidência de nascimento pré-termo ao restabelecer os níveis de hemoglobina na gestante, condição não observada na presente análise.

O uso de ferro em gestantes com níveis normais de hemoglobina também não apresentou associação com nascimento pré-termo neste trabalho, em concordância com Taylor \& Lind 30 , em estudo realizado numa maternidade britânica que comparou cinqüenta gestantes que usaram ferro e ácido fólico com cinqüenta gestantes que não utilizaram qualquer suplemento. Ensaios clínicos contraplacebo que avaliaram o desfecho nascimento pré-termo em gestantes americanas e australianas também não verificaram diferenças significativas na idade gestacional de nascimento entre os grupos 8,9,12, sugerindo que as gestantes que usaram ferro profilático durante a gravidez têm a mesma probabilidade de ter um parto prematuro que aquelas que não usaram ferro.

Em relação ao peso ao nascer, estudos observacionais anteriores verificaram uma associação protetora entre o uso de ferro e peso ao nascer 31,32, enquanto outros não 30,33,34. As discrepâncias podem ser explicadas pelas diferenças nos critérios de seleção das gestantes e na variável exposição ao ferro. Gofin et al. 33, por exemplo, compararam gestantes que usaram ferro profilático com gestantes que utilizaram ferro terapêutico. Achadi et al. 31 , por sua vez, compararam dois grupos que utilizaram diferentes quantida- des semanais de ferro, independente dos níveis de hemoglobina. Além disso, alguns estudos controlaram para fatores de confusão ${ }^{31,34}$ enquanto outros não ${ }^{33}$. Em relação aos ensaios clínicos, a maioria não verificou diferença no peso ao nascer de recém-nascidos de mães não-anêmicas que receberam ferro profilático, seja comparado com placebo 9,12 ou com esquemas posológicos diferentes 10,11.

Algumas limitações referentes aos dados utilizados nesta análise devem ser consideradas. Dados sobre os esquemas posológicos, uso de sais de ferro após a 28a semana de gestação e duração do tratamento não estavam disponíveis para análise.

Em relação à medida de hemoglobina como indicador de anemia ferropriva, cabem algumas considerações, aplicáveis não apenas a esta análise, como à grande parte dos estudos sobre intervenções na anemia durante a gestação. Embora a deficiência de ferro seja a causa mais comum de anemia na gestante, várias outras patologias podem ser responsáveis pelas alterações nos níveis normais de hemoglobina sérica, tais como malária, esquistossomose, infecções recentes ou correntes, inflamação crônica, anemias hereditárias e outras deficiências nutricionais, particularmente de ácido fólico e vitamina B12 5. Além disso, a ocorrência de expansão do volume plasmático durante a gravidez, com subseqüente hemodiluição, pode confundir a relação usual feita entre deficiência de ferro e baixos índices de hemoglobina 35 . Por isso, alguns autores defendem que o diagnóstico de anemia ferropriva deveria ser feito após a combinação do resultado da hemoglobina com o de outra medida bioquímica, como a ferritina sérica ou receptor de transferrina 1,36. Entretanto, grande parte dos estudos tem utilizado a hemoglobina como único critério diagnóstico, seguindo a definição da OMS para anemia gestacional. No presente estudo, a hemoglobina também foi adotada como único critério diagnóstico de anemia, correspondendo ao exame laboratorial preconizado pelo Ministério da Saúde para diagnóstico de anemia gestacional, considerando sua praticidade e baixo custo.

Se o uso terapêutico de sais de ferro em gestantes anêmicas parece plausível do ponto de vista biológico e epidemiológico, o mesmo não ocorre para o uso profilático. A prescrição rotineira de sais de ferro para gestantes sem diagnóstico de anemia, como medida preventiva, tem sido questionada pela falta de evidências sobre a efetividade desta prática 1,12,37. Poucos estudos, em sua maioria ensaios clínicos, avaliaram os efeitos do ferro no resultado da gestação (nascimento pré-termo e peso ao nascer). Cabe destacar que esses estudos são geralmente delineados para 
proporcionar condições ideais de utilização dos medicamentos em teste. Além disso, os estudos localizados na literatura com dados sobre desfechos clínicos relevantes foram realizados, em sua maior parte, em populações com características diferentes da população brasileira.

A alta prevalência de anemia gestacional em países em desenvolvimento, dieta pobre em alimentos contendo ferro e o baixo custo do ferro medicamentoso, em comparação com os testes necessários para o rastreamento laboratorial da anemia, constituem alguns fatores apontados para justificar a implantação de programas de suplementação a todas as gestantes, com cobertura populacional. Entretanto, outros aspectos importantes, como a adesão da paciente à profilaxia medicamentosa, devem ser considerados na análise da relação custo/benefício da profilaxia antianêmica. Os efeitos prejudiciais do ferro, que não raro causam desconforto (náuseas, vômitos, diarréia, constipação e dor estomacal), o medo da gestante de possíveis efeitos adversos relacionados com o parto e o feto, e a ausência de manifestações clínicas que comprovem o benefício do uso diário do medicamento na ausência de sintomas, são alguns fatores importantes de não-adesão ${ }^{38}$. Além disso, fatores regionais, como diferenças sócio-econômicas, prevalência de doenças como malária, composição da dieta e características genéticas que determinam os ní- veis normais de hemoglobina podem influenciar, em maior ou menor grau, os resultados de programas nacionais de suplementação de ferro.

Um outro aspecto importante relacionado à efetividade dos sais de ferro na gestação, pouco abordado na literatura, diz respeito aos benefícios clínicos para a gestante. De acordo com Makrides et al. 9, os riscos postulados da anemia ferropriva em gestantes incluem: fadiga aumentada, redução do desempenho laboral, estresse cardiovascular, resistência reduzida à infecção e baixa tolerância à perda de sangue no parto. Surpreendentemente, o possível efeito protetor da suplementação de ferro nesses desfechos raramente é investigado nos ensaios clínicos e estudos observacionais. Essa questão surge como uma importante perspectiva de pesquisa futura a ser considerada.

Em resumo, os resultados desta análise sugerem que o uso de ferro até a 28a semana de gestação por mulheres que fizeram o pré-natal em serviços vinculados ao SUS de seis capitais brasileiras não diminuiu o risco de nascimento pré-termo, baixo peso ao nascer ou muito baixo peso ao nascer. São necessários mais estudos sobre os potenciais benefícios clínicos para o recém-nascido, advindos do uso profilático e terapêutico de ferro pela gestante durante a segunda metade da gravidez.

\section{Resumo}

O objetivo foi avaliar a associação entre o uso de ferro profilático ou terapêutico com nascimento pré-termo e baixo peso ao nascer. Gestantes com vinte anos ou mais e idade gestacional entre 21 e 28 semanas foram arroladas consecutivamente em ambulatórios de prénatal ligados ao Sistema Único de Saúde (SUS) de seis capitais brasileiras entre 1991 e 1995. Características sócio-demográficas e o uso declarado de sais de ferro

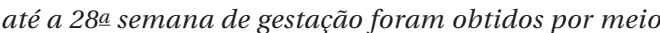
de entrevista. Os desfechos e demais variáveis foram coletados no prontuário. A prevalência de anemia entre as 3.865 gestantes analisadas foi de 31,3\%. Entre as gestantes anêmicas, 29,8\% utilizavam ferro e entre as não-anêmicas o percentual foi de 16,7\%. Após ajustamento para potenciais confundidores, o uso de ferro não mostrou associação com nascimento pré-termo ( $R C=0$, 88; IC95\%: 0,73-1,07), baixo peso ao nascer ( $R C=0,99$; IC95\%: 0,75-1,31) e muito baixo peso ao nascer ( $R C=0,58$; IC95\%: 0,29-1,13). Os resultados sugerem que o uso de ferro até a 28 a semana de gestação não diminui o risco de nascimento pré-termo, baixo peso ao nascer e muito baixo peso ao nascer.

Sais de Ferro; Gravidez; Prematuro; Peso ao Nascer 


\section{Colaboradores}

T. S. Dal Pizzol participou da revisão bibliográfica, limpeza dos dados, criação de variáveis, análise estatística, discussão dos resultados e redação do artigo. S. S. Mengue colaborou na limpeza dos dados, criação de variáveis, análise estatística e discussão dos resultados. E. R. J. Giugliani contribuiu na discussão dos resultados e revisão final do artigo.

\section{Referências}

1. Reveiz L, Gyte GML, Cuervo LG. Treatments for iron-deficiency anaemia in pregnancy (Cochrane Review). In: The Cochrane Library, Issue 3, 2007. Oxford: Update Software.

2. World Health Organization. Iron deficiency anaemia assessment, prevention, and control. Geneva: World Health Organization; 2001.

3. Ministério da Saúde. Política nacional de alimentação e nutrição. 2a Ed. Brasília: Ministério da Saúde; 2003.

4. Xiong X, Buekens P, Alexander S, Demianczuk N, Wollast E. Anemia during pregnancy and birth outcome: a meta-analysis. Am J Perinatol 2000; 17:137-46.

5. Rasmussen KM. Is there a causal relationship between iron deficiency or iron-deficiency anemia and weight at birth, lenght of gestation and perinatal mortality? J Nutr 2001; 131(2S-2):590S-601S.

6. Departamento de Ações Programáticas Estratégicas, Secretaria de Atenção à Saúde, Ministério da Saúde. Pré-natal e puerpério: atenção qualificada e humanizada. Manual técnico. Brasília: Ministério da Saúde; 2005.

7. SouzaAI, Batista Filho M, Ferreira LOC, FigueirôaJN. Efetividade de três esquemas com sulfato ferroso para tratamento de anemia em gestantes. Rev Panam Salud Pública 2004; 15: 313-9.
8. Cosgwell ME, Parvanta I, Ickes L, Yip R, Brittenham GM. Iron supplementation during pregnancy, anemia, and birth weight: a randomized controlled trial. Am J Clin Nutr 2003; 78:773-81.

9. Makrides M, Crowther CA, Gibson RA, Gibson RS, Skeaff CM. Efficacy and tolerability of low-dose iron supplements during pregnancy: a randomized controlled trial. Am J Clin Nutr 2003; 78:145-53.

10. Milman N, Bergholt T, Eriksen L, Byg KE, Graudal N, Pedersen $\mathrm{P}$, et al. Iron profilaxy during pregnancy: how much iron is needed? A randomized doseresponse study of $20-80 \mathrm{mg}$ ferrous iron daily in pregnant women. Acta Obstet Gynecol Scand 2005; 84:238-47.

11. Mukhopadhyay A, Bhatla N, Kriplani A, Pandey RM, Saxena R. Daily versus intermittent iron supplementation in pregnant women: hematological and pregnancy outcome. J Obstet Gynaecol Res 2003; 30:409-17.

12. Pena-Rosas JP, Viteri FE. Effects of routine oral iron supplementation with or without folic acid for women during pregnancy (Cochrane Review). In: The Cochrane Library, Issue 3, 2007. Oxford: Update Software.

13. Jornal Brasileiro de Medicina. Dicionário de especialidades farmacêuticas. Rio de Janeiro: Editora de Publicações Médicas; 1984. 
14. Jornal Brasileiro de Medicina. Dicionário de especialidades farmacêuticas. Rio de Janeiro: Editora de Publicações Médicas; 1985.

15. Jornal Brasileiro de Medicina. Dicionário de especialidades farmacêuticas. 15a Ed. Rio de Janeiro: Editora de Publicações Médicas; 1986.

16. Jornal Brasileiro de Medicina. Dicionário de especialidades farmacêuticas. 16a Ed. Rio de Janeiro: Editora de Publicações Médicas; 1987.

17. Jornal Brasileiro de Medicina. Dicionário de especialidades farmacêuticas. 17ạ Ed. Rio de Janeiro: Editora de Publicações Médicas; 1988.

18. Jornal Brasileiro de Medicina. Dicionário de especialidades farmacêuticas. 18a Ed. Rio de Janeiro: Editora de Publicações Médicas; 1989.

19. Jornal Brasileiro de Medicina. Dicionário de especialidades farmacêuticas 19a Ed. Rio de Janeiro: Editora de Publicações Médicas; 1990.

20. Jornal Brasileiro de Medicina. Dicionário de especialidades farmacêuticas. 20a Ed. Rio de Janeiro: Editora de Publicações Médicas; 1991.

21. Jornal Brasileiro de Medicina. Dicionário de especialidades farmacêuticas. 21ạ Ed. Rio de Janeiro: Editora de Publicações Médicas; 1992.

22. Jornal Brasileiro de Medicina. Dicionário de especialidades farmacêuticas. 22a Ed. Rio de Janeiro: Editora de Publicações Médicas; 1993.

23. Jornal Brasileiro de Medicina. Dicionário de especialidades farmacêuticas. 23a Ed. Rio de Janeiro: Editora de Publicações Médicas; 1994.

24. Jornal Brasileiro de Medicina. Dicionário de especialidades farmacêuticas. 24a ad. Rio de Janeiro: Editora de Publicações Médicas; 1995.

25. Schmidt MI, Duncan BB, Reichelt AJ, Branchtein L, Matos MC, Costa-e-Forti A, et al. Gestational diabetes mellitus diagnosed with a 2-h 75-g oral glucose tolerance test and adverse pregnancy outcomes. Diabetes Care 2001; 24:1151-5.

26. Mengue SS, Schenkel EP, Duncan BB, Schmidt MI. Uso de medicamentos por gestantes em seis cidades brasileiras. Rev Saúde Pública 2001; 35:415-20.
27. Mengue SS, Schenkel EP, Schmidt MI, Duncan BB. Fatores associados ao uso de medicamentos durante a gestação em seis cidades brasileiras. Cad Saúde Pública 2004; 20:1602-8.

28. Macgregor MW. Maternal anemia as a factor in prematurity and perinatal mortality. Scott Med J 1963; 8:134-40.

29. Hemminki E, Rimpelä U. A randomized comparison of routine versus selective iron supplementation during pregnancy. J Am Coll Nutr 1991; 10:3-10.

30. Taylor DJ, Lind T. Haematological changes during normal pregnancy: iron induced macrocytosis. $\mathrm{Br}$ J Obstet Gynaecol 1976; 83:760-7.

31. Achadi EL, Hansell MJ, Sloan NL, Anderson MA Women's nutritional status, iron consumption and weight gain during pregnancy in relation to neonatal weight and lenght in West Java, Indonesia. Int J Gynaecol Obstet 1995; 48 Suppl:S103-19.

32. Kullander S, Kallen B. A prospective study of drugs and pregnancy. Acta Obstet Gynecol Scand 1976; 55:287-95.

33. Gofin R, Adler B, Palti H. Effectiveness of iron supplementation compared to iron treatment during pregnancy. Public Health 1989; 103:139-45.

34. Knottnerus JA, Delgado LR, Knipschild PG, Essed GGM, Smits F. Haematologic parameters and pregnancy outcome. J Clin Epidemiol 1990; 43:461-6.

35. Milman N, Bergholt T, Byg KE, Eriksen L, Graudal N. Iron status and iron balance during pregnancy: a critical reappraisal of iron supplementation. Acta Obstet Gynecol Scand 1999; 78:749-57.

36. Schwartz 3rd WJ, Thurnau GR. Iron deficiency anemia in pregnancy. Clin Obstet Gynecol 1995; 38:443-54.

37. Schultnik W, Gross R. Use of daily compared with weekly iron supplementation: apples and pears. Am J Clin Nutr 1999; 69:739-42.

38. Galloway R, Dusch E, Elder L, Achadi EL, Grajeda R, Hurtado E, et al. Women's perceptions of iron deficiency and anemia prevention and control in eight developing countries. Soc Sci Med 2002; 55:529-44.

Recebido em 10/Jan/2008

Versão final reapresentada em 26/Mai/2008

Aprovado em 10/Jun/2008 